\title{
A validated quantification of triclosan in toothpaste using high-performance thin-layer chromatography and a 48-bit flatbed scanner
}

\author{
Barbara Anders $^{1} \cdot$ Sabrina Doll ${ }^{1} \cdot$ Bernd Spangenberg $^{1}$ (D
}

Received: 6 April 2021 / Accepted: 30 May 2021 / Published online: 19 June 2021

(c) The Author(s) 2021

\begin{abstract}
We present a densitometric quantification method for triclosan in toothpaste, separated by high-performance thin-layer chromatography (HPTLC) and using a 48-bit flatbed scanner as the detection system. The sample was band-wise applied to HPTLC plates $(10 \times 20 \mathrm{~cm})$, with fluorescent dye, Merck, Germany (1.05554). The plates were developed in a vertical developing chamber with $20 \mathrm{~min}$ of chamber saturation over $70 \mathrm{~mm}$, using $n$-heptane-methyl tert-butyl ether-acetic acid (92:8:0.1, V/V) as solvent. The $R_{\mathrm{F}}$ value of triclosan is $h R_{\mathrm{F}}=22.4$, and quantification is based on direct measurements using an inexpensive 48-bit flatbed scanner for color measurements (in red, green, and blue) after plate staining with 2,6-dichloroquinone-4-chloroimide (Gibbs' reagent). Evaluation of the red channel makes the measurements of triclosan very specific. For linearization, an extended Kubelka-Munk expression was used for data transformation. The range of linearity covers more than two orders of magnitude and is between 91 and $1000 \mathrm{ng}$. The separation method is inexpensive, fast and reliable.
\end{abstract}

Keywords High-performance thin-layer chromatography (HPTLC) · Thin-layer chromatography (TLC) · Triclosan .

Toothpaste $\cdot$ Flatbed scanner $\cdot$ Linear calibration range $\cdot$ Kubelka-Munk equation

\section{Introduction}

Triclosan [5-chloro-2-(2,4-dichlorophenoxy)phenol] is a polychlorinated aromatic compound (Fig. 1) and was patented in 1964 by the Swiss company Ciba-Geigy. The compound is a broad-spectrum bacteriostatic and fungicide [1]. Triclosan penetrates bacterial cells and affects the cytoplasmic membrane and synthesis of RNA, fatty acids, and proteins [2]. Triclosan has also been shown to have endocrine effects in humans and animals [3].

Five years ago, triclosan was present in a wide range of consumer product including soap, toothpaste, detergents, plastic tableware, textiles, hair products and surgical cleaning treatments. The widespread use, particularly in soaps, gave reason to discuss the risk of antimicrobial resistance with the conclusion that the risk of potential antimicrobial

Bernd Spangenberg

Spangenberg@HS-Offenburg.de

Institute of Process Engineering, Offenburg University of Applied Sciences: Hochschule Offenburg, Badstrasse 24, 77652 Offenburg, Germany resistance outweighs the benefits of the widespread use of triclosan in antimicrobial soaps [4]. Triclosan has been shown to be ubiquitous in analyzed human plasma and milk. Concentrations were shown to be higher in both plasma and milk from mothers who used personal care products containing triclosan than from mothers who did not. It has been shown that personal care products containing triclosan were one of the dominant sources of exposure to triclosan [5].

Triclosan is a widely used environmental toxin, toxic to animals and has many pharmacological effects on human population [6]. The constant high disposal of triclosan into the sewage system poses a great threat to the environment and public health [1].

The FDA (United States Food and Drug Administration) classifies triclosan as an unnecessary ingredient in antibacterial hand soaps and urges manufacturers to change their composition. In September 2016, the FDA banned the use of triclosan in soap and liquid soap in the USA. Nevertheless, other personal care products such as toothpaste, mouthwash, hand sanitizers, and surgical soaps continue to contain triclosan in high concentrations [7]. In Europe, triclosan is regulated as a cosmetic preservative and must be listed on the label. The use of triclosan in cosmetic products such as 


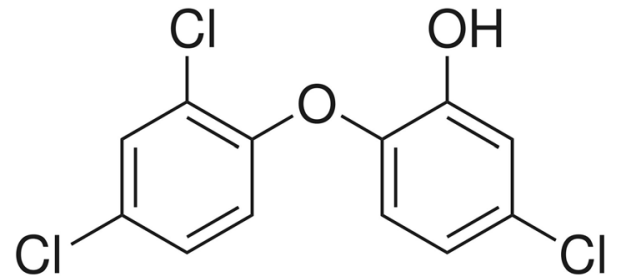

Fig. 1 The chemical structure of triclosan

toothpaste, hand soaps, body soaps, shower gels and deodorants, face powders and blemish concealers were restricted by the EU Commission in 2014 to the current maximum concentration limit of $0.3 \%$ [8]. Therefore, there is a need for an inexpensive and easy-to-perform analytical method to verify these limits in a rather complicated matrix. Highperformance thin-layer chromatography (HPTLC) should be well-suited as a quantification method in this case, since HPTLC plates are disposable and thus contaminated samples can be separated without extensive sample preparation.

A literature survey reveals that only a few papers deal with the HPTLC analysis of triclosan in toothpaste, soap or surgical products [9-12]. R. Matissek published in 1983 a TLC paper (in German) with the title: "Zur Analytik antimikrobiell wirksamer Substanzen in nicht-emulsionsartigen kosmetischen Mitteln." A rather complicated columnchromatographic pretreatment is followed here by a double development on silica gel with toluene-acetone $(4: 1, V / V)$ and then ethyl acetate-methanol-ammonia solution (10\%) $(65: 30: 5, V / V)$. TLC was used for semi-quantification of triclosan [9]. In 1999, K. Albert described the staining of triclosan on silica gel plate with 2,6-dichloroquinone4-chloroimide after an HPTLC separation with the solvent mix containing toluene-acetone $(4: 2, V / V)$ [10]. The DAC (Deutsche Arzneimittel-Codex) described this TLC method for the separation of triclosan in 2017 [11]. The publication of K. Jayaseelan and K. S. Lakshmi from 2017 describes a triclosan quantification in cosmetic products on RP-18 HPTLC plate. For quantification, the plate was scanned at $281 \mathrm{~nm}[12]$.

The purpose of this paper is to describe a rapid, simple and inexpensive working method for the quantification of triclosan in cosmetic products using HPTLC in combination with a low-cost flatbed scanner.

\section{Experimental}

\subsection{Preparation of standards and application on HPTLC plates}

All the chemicals used were of analytical reagent grade. Triclosan was purchased from Fagron $\mathrm{GmbH} \& \mathrm{Co}$. KG

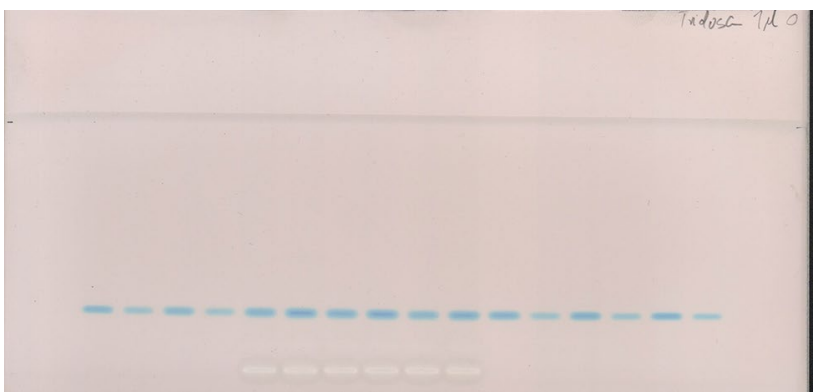

Fig. 2 Flatbed color image of a $10 \times 20 \mathrm{~cm}$ HPTLC plate with six samples (lanes 5 to 10 , counting from the left) and ten standard lanes

(Barsbüttel, Germany). Acetonitrile, $\mathrm{NaCl}, \mathrm{MgSO}_{4}, \mathrm{Na}_{2} \mathrm{CO}_{3}$, 2,6-dichloroquinone-4-chloroimide (Gibbs' reagent), methyl tert-butyl ether (MTBE), $n$-heptane, acetic acid and methanol were from Merck (Darmstadt, Germany) as well as silica gel $60 \mathrm{~F}_{254}$ plates (1.05554) with a fluorescent dye, used as stationary phase.

Stock solutions for testing linearity, content and accuracy were prepared by dissolving $5 \mathrm{mg}$ and $7 \mathrm{mg}$ triclosan in $20 \mathrm{~mL}$ of methanol (here triclosan: $4.636 \mathrm{mg}$ and $6.927 \mathrm{mg}$ ), respectively, using an Orion Cahn ${ }^{\circledR}$ C-33 microbalance from Environmental Instruments (Beverly, MA, USA). From the triclosan stock solutions, $1 \mathrm{~mL}$ was made up to $10 \mathrm{~mL}$ (working solution) having final concentrations of 231.8 and $346.35 \mathrm{ng}$ triclosan/ $\mu \mathrm{L}$. Linear calibrations from the standard deviation of the interval (taken at $\pm \sigma$ ) were used to assess the limit of detection (LOD) and limit of quantification (LOQ). To check linearity, the working solutions and 1:10 diluted working solutions were applied in amounts of 1 to $10 \mu \mathrm{L}$. Samples and standards were spotted band-wise over $7 \mathrm{~mm}$ using a CAMAG Automatic TLC Sampler (ATS 4) device (Muttenz, Switzerland). Bands were spotted at a distance of $5 \mathrm{~mm}$ from the bottom plate side and at a distance of $2 \mathrm{~cm}$ from the plate sides (Fig. 2).

\subsection{Separation, staining and spectral measurements}

For separation, silica gel plates $(10 \times 20 \mathrm{~cm})$ were developed in a vertical developing chamber at vapor saturation (20 min) to a distance of $70 \mathrm{~mm}$ from the starting point. A mixture of $n$-heptane-MTBE-acetic acid $(92: 8: 0.1, V / V)$ was used as solvent.

For staining a $10 \times 20 \mathrm{~cm}$ plate, the amount of $50 \mathrm{mg}$ 2,6-dichloroquinone-4-chloroimide was dissolved in $50 \mathrm{~mL}$ methanol using the CAMAG Derivatizer for reagent spraying (4 $\mathrm{mL}$ spray volume, blue-coded spray nozzle). In addition, the plate was sprayed with an aqueous $\mathrm{Na}_{2} \mathrm{CO}_{3}$ solution $(1 \mathrm{~g} / 10 \mathrm{~mL})$ under the same spraying conditions ( $3 \mathrm{~mL}$ spray volume, blue-coded spray nozzle). 


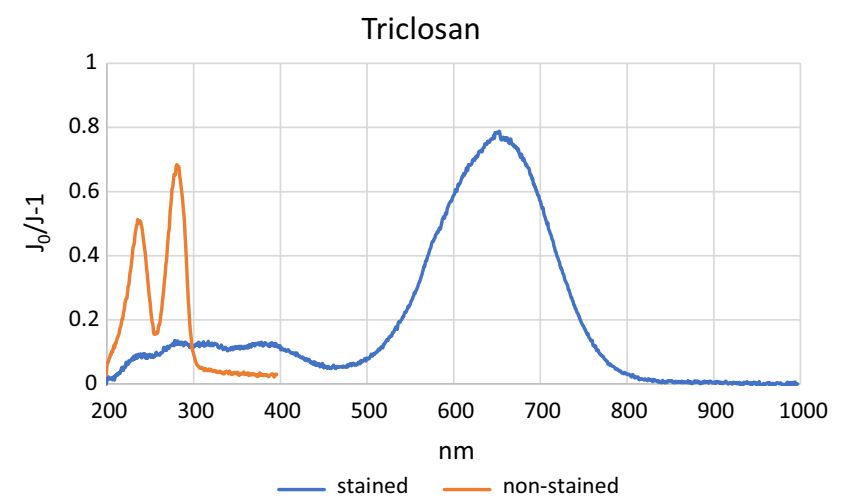

Fig. 3 UV-Vis spectra of 464 ng triclosan (non-stained) and stained with Gibbs' reagent

For spectral measurements of the unstained and stained triclosan (Fig. 3), a TIDAS TLC 2010 system from J\&M (Aalen, Germany) with a reflection attachment consisting of two light-fiber rows was used, which has a wavelength resolution of $0.8 \mathrm{~nm}$ and a spatial resolution on the plate of $100 \mu \mathrm{m}$. The measurement time for a single spectrum in the wavelength range from 190 to $1000 \mathrm{~nm}$ was $25 \mathrm{~ms}$.

For quantitative measurements of triclosan in the toothpaste Brexident ${ }^{\circledR}$ pasta dentifrice (Barcelona, Spain), the sample was prepared using the QuEChERS approach (which is the abbreviation of Quick, Easy, Cheap, Effective, Rugged and Safe). For sample preparation, an amount of $1.0 \mathrm{~g}$ toothpaste (weighted with 4 digits precision) was used and mixed with $10 \mathrm{~mL}$ of water. After vigorous shaking and sonication for $10 \mathrm{~min}$, the amount of $10 \mathrm{~mL}$ acetonitrile was added. The solution was shaken vigorously and then $1 \mathrm{~g} \mathrm{NaCl}$ and $4 \mathrm{~g} \mathrm{MgSO} 4$ were added [13]. The two phases are rapidly separated during centrifuging ( $4000 \mathrm{rpm})$, which reduces the sample extraction step to a simple centrifugation procedure. After centrifugation, the upper phase consists of $8.85 \mathrm{~mL}$ of acetonitrile. The extraction conditions are very robust. Instead of $10 \mathrm{~mL}, 5 \mathrm{~mL}$ of acetonitrile can be used to extract triclosan in the specific working range.

\subsection{Scanning with a flatbed scanner}

The digital resolution of common flatbed scanners and cameras is 8 bits. Each signal of the three color channels (red, green and blue) is converted into $2^{8}=256$ different increments, which is not sufficient for wide range quantification purposes. Here, we used a Plustek OpticSlim 500 flatbed scanner (Ahrensburg, Germany) in conjunction with the acquisition software "Presto! ImageFolio 4" [13, 14, 16]. Scanner and software allow the TLC plate to be scanned with a resolution of 16 bits for each of the three color channels, representing $2^{16}=65,536$ grey levels for each channel. The Plustek OpticSlim 500 is an inexpensive flatbed scanner and was used with a gamma factor of 1 . The short time of only $20 \mathrm{~s}$ is needed to measure the full 48-bit range of a $10 \times 20 \mathrm{~cm}$ HPTLC plate with a spatial resolution of 300 dpi. Evaluation of the stained triclosan plate was performed using the red channel and expression (1), derived from the extended Kubelka-Munk equation [13, 16].

$\mathrm{KM}=\frac{J_{0}}{J}-1=\frac{a}{1-a}$

$a$-absorption coefficient.

$J_{0}$-reflected light intensity measured from a neat plate part.

$J$-reflected light intensity measured from a track.

The self-written evaluation program ImageTLC (Ver. 5.0) is written in PureBasic (Ver. 4.50). PureBasic from Fantaisie Software (Fegersheim, France) is a commercially available 64-bit programming language based on established BASIC rules [15].

\section{Results and discussion}

HPTLC is a parallel working method where up to 16 and more samples can be applied band-wise on a single $10 \times 20 \mathrm{~cm}$ plate (see Fig. 2).

\subsection{Mobile phase selection}

For triclosan separation on silica gel 60 plates, ethyl acetate-methanol-ammonia solution (10\%) $(65: 30: 5, V / V)$ [9], toluene-acetone $(4: 1, V / V)[9]$ or toluene-acetone $(4: 2, V / V)$ [10] as well as methanol-glacial acetic acid in the ratio (7:3, $V / V)$ on RP-18 plate [12] are recommended as solvent. The DAC recommends the solvent mixture toluene-acetone $(8: 2$, $V / V)$ for separation [11]. Since all these mixtures cause more or less tailing of the triclosan peaks, we developed a new solvent mixture. A mixture of $n$-heptane-MTBE-acetic acid (92:8:0.1, $V / V)$ was the most suitable, leading to a triclosan $R_{\mathrm{F}}$ value of $h R_{\mathrm{F}}=22.4$. The small amount of acetic acid avoids peak-tailing. Formic acid can also be used instead of acetic acid. Separation conditions are robust, as mixtures of 90\% $n$-heptane with $10 \%$ MTBE up to $95 \% n$-heptane with 5\% MTBE also adequately separate triclosan from all excipients. Due to the large separation chambers for $10 \times 20 \mathrm{~cm}$ HPTLC plates, vapor saturation of $20 \mathrm{~min}$ is recommended but not necessary. Without vapor saturation, the $R_{\mathrm{F}}$ value of triclosan is not stable, and therefore all bands for calculation must be on a single plate. Identical amounts of triclosan may differ from plate to plate, but in the case of vapor saturation, even values from two different plates can be used in combination without introducing a large error. 


\subsection{TLC plate-staining using Gibbs' reagent}

Triclosan shows absorption maxima at 242 and around $281 \mathrm{~nm}$ and cannot be detected by a Vis scanner. The reagent 2,6-dichloroquinone-4-chloroimide (Gibbs' reagent) was used for staining because the chloroimide reacts rapidly at room temperature with all phenolic compounds unsubstituted in para position to form a blue dye at high $\mathrm{pH}$ values [13]. Also reacting are $\mathrm{N}$-containing heterocyclic compounds, indoles, carbazoles, coumarins, barbiturates, and capsaicin and phenoxyacetic acid [17, 18]. Triclosan reacts with Gibbs' reagent within seconds at room temperature [10, 11]. The absorption signal of the blue dye ranges from 550 to $750 \mathrm{~nm}$. Its absorption maximum is at $650 \mathrm{~nm}$ (Fig. 3.)

\subsection{TLC plate-scanning using a flatbed scanner}

After sample application and separation, the plate is illuminated with light of different wavelength $J_{o}(\lambda)$. This light is scattered and absorbed by both the analyte and the layer and can be detected as reflected light $J(\lambda)$ from above the layer. For the detection of light in the visible range (Vis range), the easiest way to perform track evaluations is with a CCD camera or a flatbed scanner. Flatbed scanners are sensitive to light in three wavelength ranges $(\lambda)$, as the plate is illuminated by blue light (420-495 nm), green light (495-570 nm), and orange/red light $(570-740 \mathrm{~nm})$. According to Fig. 3, stained triclosan is weakly detectable in the green channel and strongly detectable in the red channel. The problem with plate scanning is that the illuminating light intensity $J_{o}(\lambda)$ must be constant over the entire plate surface. This is the reason why we prefer flatbed scanners to cameras, because they not only have a high spatial resolution but also show a constant light illumination on the plate.

After measuring $J$ and $J_{0}$, the data must be transformed to obtain a linear calibration function so that simple quantification calculations can be performed. Theoretical considerations lead to Eq. (1), which is valid in the ultraviolet-visible (UV-Vis) range for all scanner systems [13].

\subsection{Linear calibration function in HPTLC}

The quantitative analysis of triclosan is possible in the range between the limit of quantification (LOQ) and the largest value of the calibration range. For this purpose, LOD (limit of detection) and LOQ must be determined, which is preferably done by constructing a calibration curve with a confidence interval of $\pm 3 \sigma$ (significance level of $\alpha=0.002$ ) in a range from 10 to $1000 \mathrm{ng} / \mathrm{band}$ (see Fig. 4). The LOD was calculated as $3 \sigma$ and LOQ as $9 \sigma$, where $\sigma$ is the standard deviation of the calibration function at a significance level of $\alpha=0.002$ (see Fig. 4) [13, 16].

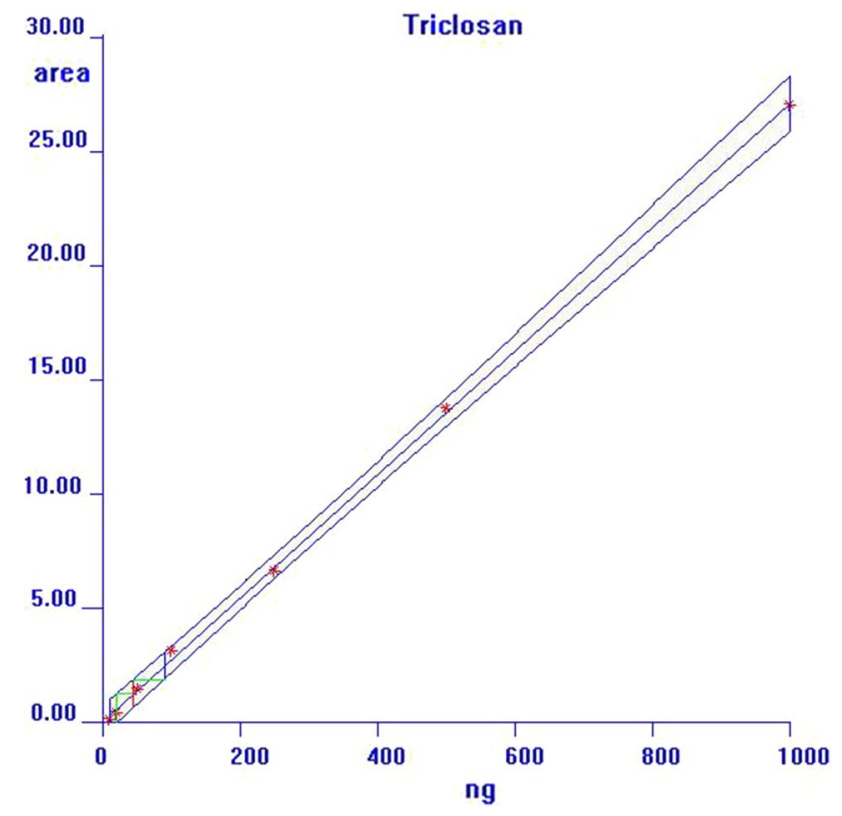

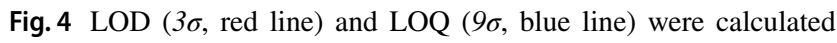
with the standard deviation $\sigma$ of the calibration function, calculated at a significance level of $\alpha=0.002$

According to Fig. 4, the LOD of triclosan was calculated to be $46 \mathrm{ng}$ and the LOQ was calculated to be $91 \mathrm{ng}$ per band. Thus, the analytical range of the triclosan quantification method is $91-1000 \mathrm{ng} / \mathrm{band}$. A linear calibration graph with zero intercept is suitable for quantification because the simple rule of proportion can be used, so linear calibration is not recommended.

\subsection{Sample pre-treatment (QuEChERS extraction)}

The QuEChERS extraction method was used for the pretreatment of the rather complex toothpaste formulation. The paste in an amount of $1.000 \mathrm{~g}$ is uniformly suspended in a mixture of water $(10 \mathrm{~mL})$ to which acetonitrile $(10 \mathrm{~mL})$ is subsequently added. Here, six amounts of toothpaste around $1 \mathrm{~g}$ were weighted with an accuracy of 4 digits and extracted. The procedure allows quick and easy extraction of suspensions in combined with rapid phase separation. The preparation for six samples is completed within $30 \mathrm{~min}$.

Two pitfalls of the QuEChERS extraction method should be mentioned. Adjustable air displacement pipettes with disposal tips are calibrated for water. A test with a 5-mL pipette showed high precision and accuracy, but this does not hold for pipetting acetonitrile. Pipetting 5-mL acetonitrile using an air displacement pipette resulted in an amount of $4.89 \mathrm{~mL}$, which corresponds to an error of $2.2 \%$. This was verified by using a fine burette $(10 \mathrm{~mL}$, Brandt, Germany); therefore, acetonitrile should be added with such a fine burette! 
A second problem is the residual amount of acetonitrile after phase separation. During a QuEChERS extraction, two phases are formed from a homogeneous mixture of sample and extractant by adding a defined salt amount [13]. The dissolved salt displaces the organic phase from the water forming a new organic phase. Depending on the type of the sample, the amount of the organic phase varies and must be determined. Here, the amount of $10 \mathrm{~mL} 0.3 \%$ triclosan solution in $\mathrm{CH}_{3} \mathrm{CN}$ was mixed with the same amount of water in which $1 \mathrm{~g}$ of toothpaste (without triclosan, but containing a surfactant) was suspended. After salt addition and phase separation, the content of triclosan in the organic phase of two preparations was measured and compared with the $0.3 \%$ triclosan solution. The triclosan concentration in the organic extract was increased by $13.0 \%$ in comparison with the original triclosan solution, so the organic phase consisted of $8.85 \mathrm{~mL}$ rather than $10 \mathrm{~mL}$.

\subsection{Precision of triclosan in toothpaste}

Six toothpaste samples were prepared according to QuEChERS extraction conditions. After extraction, $2 \mu \mathrm{L}$ sample extracts were applied band-wise $(7 \mathrm{~mm})$ onto a $10 \times 20 \mathrm{~cm}$ plate, in addition to ten tracks of two different standard solutions. After separation and staining, the plate was scanned using the red channel of the flatbed scanner. An image of the plate is shown in Fig. 2. The TIF image was evaluated track by track by bundling 50 diodes into a single densitogram from which the peak areas of the triclosan signals were extracted (Fig. 5). All data are shown in Table 1.

The variance of the sample is $\operatorname{var}\left(y_{a}\right)=0.2507$ and of the standard is $\operatorname{var}\left(y_{s}\right)=0.2351$. According to the so-called rule of three (2), which describes a simple procedure for solving a linear equation (without intercept!), the content

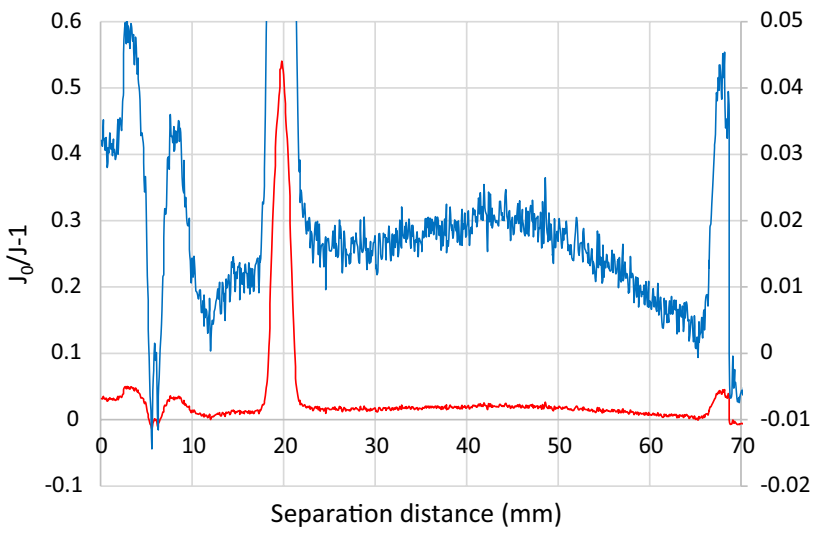

Fig. 5 Densitogram of a triclosan separation from a toothpaste sample (blue: densitogram increased tenfold)

of the analyte can be calculated from the mean values of analyte and standard measurements (3).

$\frac{a \overline{X_{a}}}{a \overline{X_{s}}}=\frac{\overline{Y_{a}}}{\overline{Y_{s}}} \frac{1}{2 * 1.13 * 1.0088}$

$\overline{X_{a}}=0.4386 * \overline{X_{s}} \frac{\overline{Y_{a}}}{\overline{Y_{s}}}$

The measurement of both means (analyte and standard) contribute to the overall uncertainty of the final result, so both measurements must be considered when calculating the overall uncertainty of the final result. The confidence interval is calculated according to Eq. (4) for $n=6$ analyte and $m=10$ standard measurements with $t(n, m)$ the appropriate Student factors for a significance level of $\alpha=0.05$.
Table 1 Analytical data from six weighted toothpaste samples and ten triclosan standard measurements

\begin{tabular}{|c|c|c|c|c|c|}
\hline \multirow{2}{*}{$\begin{array}{l}\text { Weight of } \\
\text { toothpaste }(\mathrm{g})\end{array}$} & \multicolumn{3}{|c|}{ Sample ( $2 \mu \mathrm{L}$ applied $)$} & \multicolumn{2}{|c|}{ Triclosan (standard, $2 \mu \mathrm{L}$} \\
\hline & Area & Corrected area & Standard (ng) & Area & Corrected area \\
\hline 1.0104 & 7.0284 & 7.0175 & 692.7 & 10.724 & 8.9506 \\
\hline 0.9602 & 7.5368 & 7.9185 & 463.6 & 6.290 & 7.8442 \\
\hline 0.9699 & 7.9803 & 8.3006 & 692.7 & 10.693 & 8.9247 \\
\hline 1.0562 & 8.7648 & 8.3717 & 463.6 & 6.359 & 7.9302 \\
\hline 1.0674 & 8.0034 & 7.5643 & 692.7 & 9.784 & 8.1660 \\
\hline \multirow[t]{5}{*}{0.9889} & 7.6655 & 7.8200 & 463.6 & 6.092 & 7.5973 \\
\hline & & & 692.7 & 10.113 & 8.4406 \\
\hline & & & 463.6 & 6.298 & 7.8542 \\
\hline & & & 692.7 & 10.440 & 8.7136 \\
\hline & & & 463.6 & 6.4153 & 8.0004 \\
\hline \multirow[t]{2}{*}{1.0088} & $Y a=7.8299$ & & $X s=578.15$ & $Y s=8.321$ & \\
\hline & $\operatorname{var}(y a)=0.2507$ & & & $\operatorname{var}(y s)=0.2351$ & \\
\hline
\end{tabular}


$\operatorname{cnf}\left(\overline{X_{y}}, t\right)=\overline{X_{a}} \pm \overline{X_{a}} \sqrt{\left(\operatorname{var}\left(y_{a}\right) \frac{t_{n-1}^{2}}{n \bar{Y}_{a}^{2}}+\operatorname{var}\left(y_{s}\right) \frac{t_{m-1}^{2}}{m \bar{Y}_{s}^{2}}\right)}$

The measurement data (Table 1) in combination with Eqs. (3) and (4), the final concentration of triclosan in toothpaste is $238.6 \pm 18.85 \mathrm{ng}$ triclosan per $\mathrm{g}$ toothpaste. This is an overall analytical error of $7.9 \%$.

\subsection{Specificity of triclosan in toothpaste}

The method is specific for triclosan, since no other compound in the toothpaste is stained by Gibbs' reagent. The symmetrical peak shape of stained triclosan (track 5 in Fig. 2) is shown in Fig. 5 and also gives an indication that the triclosan peak is not contaminated by other compounds.

\subsection{Accuracy of triclosan in toothpaste}

To verify the accuracy of the method, the amount of $30 \mathrm{~g}$ of triclosan $\left(30.81 \mathrm{mg}\right.$ ) was dissolved in $20 \mathrm{~mL}$ of $\mathrm{CH}_{3} \mathrm{CN}$. Six weights of toothpaste $(1.000 \mathrm{~g})$ were made up with $1 \mathrm{~mL}$ each of this triclosan standard. Acetonitrile was removed by a gentle air flow. After QuEChERS extraction, the extracts were applied to the plate (in the amount of $2 \mu \mathrm{L}$ ), separated, stained, and analyzed as described. The measurement data are shown in Table 2.

According to Table 2 in combination with Eqs. (4) and (5), the final standard addition concentration of triclosan in toothpaste is $365.8 \pm 35.0 \mathrm{ng}$ triclosan per $\mathrm{g}$ toothpaste. This corresponds to an overall analytical error of $9.56 \%$.

$\overline{X_{a}}=0.4204 * \overline{X_{s}} \frac{\overline{Y_{a}}}{\overline{Y_{s}}}$

The recovery rate is $93.2 \%$. Thus, the method can be considered accurate.

\subsection{Robustness of the method}

The method is robust because the solvent composition can vary in a wide range without affecting the results. Triclosan from $1 \mathrm{~g}$ of toothpaste can be extracted with 5-10 $\mathrm{mL}$ acetonitrile and the staining process will only convert triclosan in a blue colored compound. All compound that could influence the triclosan detection remain at the application point or migrate in the front, like the surfactants the toothpaste contain, as shown in Figs. 2 and 5.

\section{Conclusions}

HPTLC is a cost-effective and easy-to-use separation technique suitable for monitoring cosmetic articles. The presented HPTLC method is well-suited to quantify triclosan in the complex matrix of toothpaste with a simple pre-treatment procedure. The working range is quite wide, ranging from 91 to $1000 \mathrm{ng}$ per band. The overall uncertainty of triclosan quantification for six independent work-ups is less
Table 2 Analytical data from ten standard triclosan measurements and six toothpaste samples, each with a standard addition of $1.5405 \mathrm{mg}$ triclosan per $\mathrm{g}$ toothpaste

\begin{tabular}{|c|c|c|c|c|c|}
\hline \multirow{2}{*}{$\begin{array}{l}\text { Toothpaste }(\mathrm{g}) \\
\text { Standard addition: } \\
1.5405 \mathrm{mg} \text { triclosan }\end{array}$} & \multicolumn{3}{|c|}{ Sample ( $2 \mu \mathrm{L}$ applied) } & \multicolumn{2}{|c|}{ Triclosan (standard, $2 \mu \mathrm{L}$ ) } \\
\hline & Area & Corrected area & Standard (ng) & Area & Corrected area \\
\hline 0.9696 & 11.729 & 12.901 & 692.7 & 10.001 & 8.3472 \\
\hline 1.1839 & 14.818 & 13.348 & 463.6 & 6.3081 & 7.8668 \\
\hline 0.9753 & 12.491 & 13.658 & 692.7 & 10.168 & 8.4865 \\
\hline 1.1144 & 14.106 & 13.499 & 463.6 & 6.1689 & 7.6932 \\
\hline 1.1086 & 10.997 & 10.579 & 692.7 & 11.152 & 9.3078 \\
\hline \multirow[t]{5}{*}{1.0469} & 13.043 & 13.287 & 463.6 & 6.3185 & 7.8797 \\
\hline & & & 692.7 & 11.085 & 9.2519 \\
\hline & & & 463.6 & 6.2126 & 7.7477 \\
\hline & & & 692.7 & 10.952 & 9.1409 \\
\hline & & & 463.6 & 6.0416 & 7.5344 \\
\hline \multirow[t]{2}{*}{1.0526} & $Y a=12.7019$ & & $X s=578.15$ & $Y s=8.441$ & \\
\hline & $\operatorname{var}(y a)=1.0829$ & & & $\operatorname{var}(y s)=0.4759$ & \\
\hline
\end{tabular}

than $8 \%$. The six samples can we weighed and extracted 
within $40 \mathrm{~min}$, and sample application is completed in $6 \mathrm{~min}$. The separation requires $12 \mathrm{~min}$, staining $5 \mathrm{~min}$ and plate scanning $30 \mathrm{~s}$. Taking drying times into account, six samples can be analyzed in a maximum of $90 \mathrm{~min}$. This is clearly superior to column chromatographic separation methods.

Compared to sophisticate and elaborate analytical systems, the presented method is easy to perform and does not require sophisticated equipment. The scanner used is compact and easy to transport, although the data quality is comparable to much more expensive TLC scanners, as the densitogram in Fig. 5 clearly shows. The 48-bit flatbed scanner makes HPTLC a cost-effective method that could also be applicable in less developed countries.

In conclusion, a 48-bit flatbed scanner is a truly high-tech tool. It is commercially available at a very low price $(105 €)$ and makes HPTLC analysis a fully quantitative method without compromising its simplicity.

Acknowledgement The authors express their appreciation to Merck Company (Darmstadt, Germany) and BioStep (Burkhardtsdorf, Germany) for their kind support.

Funding Open Access funding enabled and organized by Projekt DEAL.

Open Access This article is licensed under a Creative Commons Attribution 4.0 International License, which permits use, sharing, adaptation, distribution and reproduction in any medium or format, as long as you give appropriate credit to the original author(s) and the source, provide a link to the Creative Commons licence, and indicate if changes were made. The images or other third party material in this article are included in the article's Creative Commons licence, unless indicated otherwise in a credit line to the material. If material is not included in the article's Creative Commons licence and your intended use is not permitted by statutory regulation or exceeds the permitted use, you will need to obtain permission directly from the copyright holder. To view a copy of this licence, visit http://creativecommons.org/licenses/by/4.0/.

\section{References}

1. Halden RU (2014) On the need and speed of regulating triclosan and triclocarban in the United States. Environ Sci Technol 48(7):3603-3611. https://doi.org/10.1021/es500495p.PMC39 74611.PMID24588513

2. Jones RD, Jampani HB, Newman JL, Lee AS (2000) Triclosan: a review of effectiveness and safety in health care settings. Am J
Infect Control 28(2):184-196. https://doi.org/10.1067/mic.2000. 102378

3. Jung EM, An BS, Choi KC, Jeung EB (2012) Potential estrogenic activity of triclosan in the uterus of immature rats and rat pituitary GH3 cells. Toxicol Lett 208(2):142-148. https://doi.org/10.1016/j. toxlet.2011.10.017

4. Giuliano CA, Rybak MJ (2015) Efficacy of triclosan as an antimicrobial hand soap and its potential impact on antimicrobial resistance: a focused review. Pharmacotherapy 35(3):328-336. https:// doi.org/10.1002/phar.1553,PMID25809180

5. Allmyr M, Adolfsson-Erici M, McLachlan MS, SandborghEnglund G (2006) Triclosan in plasma and milk from Swedish nursing mothers and their exposure via personal care products. Sci Total Environ 372(1):87-93. https://doi.org/10.1016/j.scito tenv.2006.08.007

6. Yueh MF, Tukey RH (2016) Triclosan: a widespread environmental toxicant with many bbiological effects. Annu Rev Pharmacol Toxicol 56:251-272. https://doi.org/10.1146/annurev-pharm tox-010715-103417

7. Weatherly LM, Gosse JA (2017) Triclosan exposure, transformation, and human health effects. J Toxicol Environ Healtht B 20:S447-S469. https://doi.org/10.1080/10937404.2017.1399306.

8. Commission Regulation (EU) No 358/2014 of 9 April 2014, https://eur-lex.europa.eu/eli/reg/2014/358/oj

9. Matissek R (1983) Zur Analytik antimikrobiell wirksamer Substanzen in nicht-emulsionsartigen kosmetischen Mitteln. Z Lebensm Unters Forsch 176(2):95-101. https://doi.org/10.1007/ BF01074470

10. Albert K (1999) Identitätsprüfung von triclosan. Pharmazeutische Zeitung 16. https://www.pharmazeutische-zeitung.de/inhalt-161999/pharm7-16-1999/

11. Deutsche Arzneimittel-Codex (DAC) Triclosan 2017-2, T-125, 274

12. Jayaseelan K, Lakshmi KS (2017) Central composite design to develop a robust RP-TLC/densitometry method for quantification of triclosan in cosmetic preparations. Marmara Pharmaceutical J 21(3):612-619. https://doi.org/10.12991/marupj.323289

13. Spangenberg B, Poole CF, Weins C (2010) Quantitative thin-layer chromatography. A practical survey. Springer, Berlin

14. http://plustek.com/usa/products/personal-desktop-scanners/optic slim-500-plus/. Retrieved 18 March 2021

15. Official website of PureBasic (2021) https://www.purebasic.com/. Retrieved 18 March 2021

16. Spangenberg B, Weyandt-Spangenberg M (2016) A validated quantification of methadone in liquids using thin-layer chromatography and a flatbed scanner. J Planar Chromatogr-Mod TLC 29(1):59-65. https://doi.org/10.1556/1006.2016.29.1.7

17. Jork H, Funk W, Fischer W, Wimmer H (1990) Thin-Layer Chromatography: Reagents and Detection Methods, vol 1a. VCH, Weinheim

18. Jork H, Funk W, Fischer W, Wimmer H (1994) Thin-layer chromatography: reagents and detection methods, vol $1 \mathrm{~b}$. VCH, Weinheim 